\title{
A COMMENT ON FREE GROUP FACTORS
}

\author{
NARUTAKA OZAWA \\ Department of Mathematical Sciences, University of Tokyo \\ Tokyo 153-8914, Japan \\ E-mail: narutaka@ms.u-tokyo.ac.jp
}

\begin{abstract}
Let $M$ be a finite von Neumann algebra acting on the standard Hilbert space $L^{2}(M)$. We look at the space of those bounded operators on $L^{2}(M)$ that are compact as operators from $M$ into $L^{2}(M)$. The case where $M$ is the free group factor is particularly interesting.
\end{abstract}

1. Introduction. In the paper $\mathrm{Oz} 1$, it is proved that the free group factor $\mathcal{L} \mathbb{F}_{r}$ is solid, i.e., the relative commutant $B^{\prime} \cap \mathcal{L} \mathbb{F}_{r}$ of any diffuse subalgebra $B$ is amenable. The proof relies on $\mathrm{C}^{*}$-algebra techniques. (See $\mathrm{Pe}, \mathrm{Po} 2$ for purely von Neumann algebraic proofs of this fact.) In particular, the crucial ingredient in Oz1 is Akemann and Ostrand's theorem $([\mathrm{AO}]$ ) stating that the $*$-homomorphism

$$
\mu: C_{\lambda}^{*} \mathbb{F}_{r} \otimes_{\text {alg }} C_{\rho}^{*} \mathbb{F}_{r} \ni \sum a_{k} \otimes x_{k} \mapsto \sum a_{k} x_{k}+\mathbb{K}\left(\ell^{2} \mathbb{F}_{r}\right) \in \mathbb{B}\left(\ell^{2} \mathbb{F}_{r}\right) / \mathbb{K}\left(\ell^{2} \mathbb{F}_{r}\right)
$$

is continuous w.r.t. the minimal tensor norm. It would be interesting to know how much of the proof in Oz1 can be carried out at the level of von Neumann algebras. In this paper, we will prove a version of Akemann and Ostrand's theorem in the von Neumann setting. For this purpose, we consider the set of those operators in $\mathbb{B}\left(L^{2}(M)\right)$ that are compact as operators from $M$ into $L^{2}(M)$, where $M=\mathcal{L} \mathbb{F}_{r}$ or any finite von Neumann algebra.

2. Compact operators. Let $\mathcal{H}$ be a Hilbert space. We denote by $\mathbb{B}(\mathcal{H})($ resp. $\mathbb{K}(\mathcal{H}))$ the $\mathrm{C}^{*}$-algebra of all bounded (resp. compact) linear operators on $\mathcal{H}$. Let $\Omega \subset \mathcal{H}$ be a closed balanced bounded convex subset. (Recall that $\Omega$ is said to be balanced if $\alpha \Omega \subset \Omega$ for $\alpha \in \mathbb{C}$ with $|\alpha| \leq 1$.) We define the closed left ideal $\mathbb{K}_{\Omega}^{L}$ of $\mathbb{B}(\mathcal{H})$ by

$$
\mathbb{K}_{\Omega}^{L}=\{x \in \mathbb{B}(\mathcal{H}): x \Omega \text { is norm compact in } \mathcal{H}\} \text {. }
$$

2000 Mathematics Subject Classification: Primary 46L10; Secondary 46L07.

Key words and phrases: von Neumann algebras, free group factors.

The paper is in final form and no version of it will be published elsewhere. 
We define a seminorm $\|\cdot\|_{\Omega}$ on $\mathbb{B}(\mathcal{H})$ by

$$
\|x\|_{\Omega}=\sup \{\|x \xi\|: \xi \in \Omega\} .
$$

We will use the following trivial proposition without quoting it.

Proposition. Let $\Omega \subset \mathcal{H}$ be as above. Then, for $x \in \mathbb{B}(\mathcal{H})$, the following are equivalent.

1. $x \in \mathbb{K}_{\Omega}^{L}$.

2. $x$ is weak-norm continuous on $\Omega$.

3. For every weakly null sequence $\left(\xi_{n}\right)$ in $\Omega$, one has $\left\|x \xi_{n}\right\| \rightarrow 0$.

4. For every sequence of (finite-rank) projections $\left(Q_{n}\right)$ strongly converging to 1 on $\mathcal{H}$, one has $\left\|x-Q_{n} x\right\|_{\Omega} \rightarrow 0$.

5. There exists a sequence $\left(x_{n}\right)$ in $\mathbb{K}(\mathcal{H})$ such that $\left\|x-x_{n}\right\|_{\Omega} \rightarrow 0$.

Definition. We denote by $\mathbb{K}_{\Omega}$ the hereditary $\mathrm{C}^{*}$-subalgebra of $\mathbb{B}(\mathcal{H})$ associated with the left ideal $\mathbb{K}_{\Omega}^{L}$ :

$$
\mathbb{K}_{\Omega}=\left(\mathbb{K}_{\Omega}^{L}\right)^{*} \cap \mathbb{K}_{\Omega}^{L}=\left(\mathbb{K}_{\Omega}^{L}\right)^{*} \cdot \mathbb{K}_{\Omega}^{L}
$$

Let $x \in \mathbb{K}_{\Omega}$. For finite-rank projections $Q_{n} \nearrow 1$ on $\mathcal{H}$, we define $x_{n}=a Q_{n} b$, where $b=|x|^{1 / 2}$ and $a=x b^{-1}$ are in $\mathbb{K}_{\Omega}$. Then, $x_{n} \in \mathbb{K}(\mathcal{H})$ satisfies $\left\|x_{n}\right\| \leq\|x\|$ and $\| x-$ $x_{n}\left\|_{\Omega}+\right\| x^{*}-x_{n}^{*} \|_{\Omega} \rightarrow 0$.

3. Finite von Neumann algebras. Let $M$ be a finite von Neumann algebra with a distinguished faithful normal trace $\tau$, and $L^{2}(M)$ be the GNS-Hilbert space associated with $(M, \tau)$. We will write $\hat{a}$ for $a \in M$ when viewed as a vector in $L^{2}(M)$, and $\|a\|_{2}=$ $\|\hat{a}\|=\tau\left(a^{*} a\right)^{1 / 2}$. From now on, we set

$$
\Omega=\{\hat{a}: a \in M,\|a\| \leq 1\} \subset L^{2}(M)
$$

and write $\mathbb{K}_{M}$ instead of $\mathbb{K}_{\Omega}$. It is clear that both $M$ and $M^{\prime}$ are in the multiplier of $\mathbb{K}_{M}$. The $\mathrm{C}^{*}$-algebra $\mathbb{K}_{M}$ is much larger than $\mathbb{K}\left(L^{2}(M)\right)$. Indeed, if $p_{n}$ are mutually orthogonal projections in $M$ (or in $M^{\prime}$ ) and $x_{n}$ are compact contractive operators such that $x_{n}=p_{n} x_{n} p_{n}$, then $\sum x_{n} \in \mathbb{K}_{M}$. The following is useful in understanding the nature of the norm $\|\cdot\|_{\Omega}$.

Lemma. For every $x \in \mathbb{B}(\mathcal{H})$, one has

$$
\|x\|_{\Omega} \leq \inf \left\{\|y\|\|b\|_{2}+\|z\|\left\|c^{\prime}\right\|_{2}\right\} \leq 4\|x\|_{\Omega},
$$

where the infimum is taken over all possible decompositions $x=y b+z c^{\prime}$ with $y, z \in \mathbb{B}(\mathcal{H})$, $b \in M$ and $c^{\prime} \in M^{\prime}$.

Proof. Since

$$
\|y b\|_{\Omega}=\sup _{a \in(M)_{1}}\|y b \hat{a}\| \leq\|y\| \sup _{a \in(M)_{1}}\|\widehat{b a}\| \leq\|y\| \sup _{a \in(M)_{1}}\|\hat{b}\|\|a\|=\|y\|\|b\|_{2}
$$

and $\left\|z c^{\prime}\right\|_{\Omega} \leq\|z\|\left\|c^{\prime}\right\|_{2}$ similarly, one has $\left\|y b+z c^{\prime}\right\|_{\Omega} \leq\|y\|\|b\|_{2}+\|z\|\left\|c^{\prime}\right\|_{2}$.

To prove the other inequality, let $x \in \mathbb{B}(\mathcal{H})$ be given such that $\|x\|_{\Omega}=1$. We observe that $\|x\|_{\Omega}$ is nothing but the norm as an operator from $M$ into $L^{2}(M)$. It follows from the noncommutative little Grothendieck inequality (Theorem 9.4 in [Pi]) that there are unit vectors $\zeta, \eta \in L^{2}(M)$ such that $\|x \hat{a}\|^{2} \leq\|a \zeta\|^{2}+\|\eta a\|^{2}$ for all $a \in M$. We view $\zeta$ and 
$\eta$ as square integrable operators affiliated with $M$ (see Appendix $\mathrm{F}$ in [BO] or Chapter IX in [Ta] $)$, and let $q=\chi_{\left(\|x\|^{2}, \infty\right)}\left(\zeta \zeta^{*}\right), p=\chi_{\left(\|x\|^{2}, \infty\right)}\left(\eta^{*} \eta\right)$. It follows that

$$
\begin{aligned}
\|x \hat{a}\|^{2} & \leq 2\left(\left\|x\left(p^{\perp} \hat{a} q^{\perp}\right)\right\|^{2}+\left\|x\left(p \hat{a} q^{\perp}\right)\right\|^{2}+\|x(\hat{a} q)\|^{2}\right) \\
& \leq 2\left(\left\|a q^{\perp} \zeta\right\|_{2}^{2}+\left\|\eta p^{\perp} a\right\|_{2}^{2}+\|x\|^{2}\|p a\|_{2}^{2}+\|x\|^{2}\|a q\|_{2}^{2}\right) \\
& =2\left(\|b \hat{a}\|_{2}^{2}+\left\|c^{\prime} \hat{a}\right\|_{2}^{2}\right),
\end{aligned}
$$

where $b$ is the left multiplication operator by $\left(p^{\perp} \eta^{*} \eta p^{\perp}+\|x\|^{2} p\right)^{1 / 2}$ and $c^{\prime}$ is the right multiplication operator by $\left(q^{\perp} \zeta \zeta^{*} q^{\perp}+\|x\|^{2} q\right)^{1 / 2}$. Note that one has $b \in M,\|b\| \leq\|x\|$ and $\|b\|_{2} \leq\|\zeta\|_{2}=1$; and likewise for $c^{\prime} \in M^{\prime}$. It follows that there are operators $y, z \in \mathbb{B}(\mathcal{H})$ with $y y^{*}+z z^{*} \leq 2$ such that $x=y b+z c^{\prime}$.

The "cb-version" of the norm $\|\cdot\|_{\Omega}$ is defined to be

$$
\begin{aligned}
\|x\|_{\tau} & =\sup \left\{\left(\sum\left\|x \hat{a}_{n}\right\|^{2}\right)^{1 / 2}:\left(a_{n}\right)_{n=1}^{\infty} \in M \text { such that } \sum a_{n} a_{n}^{*} \leq 1\right\} \\
& =\left\|x: M^{\prime} \ni a^{\prime} \mapsto x a^{\prime} \hat{1} \in L^{2}(M)_{\mathrm{col}}\right\|_{\mathrm{cb}} \\
& =\inf \left\{\|y\|\|b\|_{2}: y \in \mathbb{B}(\mathcal{H}) \text { and } b \in M \text { with } x=y b\right\} .
\end{aligned}
$$

We do not elaborate on this norm here. See [Ma] for more information about the topology associated with this norm.

4. Free group factors. We write $\lambda$ and $\rho$ respectively for the left and the right regular representation of a countable discrete group $\Gamma$ on $\ell^{2} \Gamma$. Recall that the group $\Gamma$ is in the class $\mathcal{S}$ if it is exact and the $*$-homomorphism

$$
\mu: C_{\lambda}^{*} \Gamma \otimes_{\text {alg }} C_{\rho}^{*} \Gamma \ni \sum a_{k} \otimes x_{k} \mapsto \sum a_{k} x_{k}+\mathbb{K}\left(\ell^{2} \Gamma\right) \in \mathbb{B}\left(\ell^{2} \Gamma\right) / \mathbb{K}\left(\ell^{2} \Gamma\right)
$$

is continuous w.r.t. the minimal tensor norm. Free groups as well as hyperbolic groups are in the class $\mathcal{S}$. (See Oz2.) Let $\Gamma$ be an ICC group so that the group von Neumann algebra $\mathcal{L} \Gamma=\lambda(\Gamma)^{\prime \prime} \subset \mathbb{B}\left(\ell^{2} \Gamma\right)$ is a factor. We note that $L^{2}(\mathcal{L} \Gamma)$ is canonically isomorphic to $\ell^{2} \Gamma$. We denote $\mathcal{R} \Gamma=(\rho(\Gamma))^{\prime \prime}=(\mathcal{L} \Gamma)^{\prime}$ and consider the $*$-homomorphism

$$
\pi: C^{*}(\mathcal{L} \Gamma, \mathcal{R} \Gamma) \ni \sum a_{k} x_{k} \mapsto \sum a_{k} \otimes x_{k} \in \mathcal{L} \Gamma \otimes_{\min } \mathcal{R} \Gamma \subset \mathbb{B}\left(\ell^{2} \Gamma \otimes \ell^{2} \Gamma\right),
$$

which is well-defined by Takesaki's theorem on the minimal tensor norm. The following theorem extends Akemann and Ostrand's theorem ([AO]).

THEOREM. Let $\Gamma$ be an ICC group which is in the class $\mathcal{S}$. Then

$$
\operatorname{ker} \pi=\mathbb{K}_{\mathcal{L} \Gamma} \cap C^{*}(\mathcal{L} \Gamma, \mathcal{R} \Gamma) \text {. }
$$

Proof. Take any sequence $\left(\xi_{n}\right)$ of unit vectors in $\Omega=\{\hat{a}: a \in \mathcal{L} \Gamma,\|a\| \leq 1\}$, which weakly converges to 0 . We define a state $\omega$ on $C^{*}(\mathcal{L} \Gamma, \mathcal{R} \Gamma)$ by the Banach limit $\omega(x)=$ $\operatorname{Lim}\left\langle x \xi_{n}, \xi_{n}\right\rangle$. Let $y \in \operatorname{alg}(\mathcal{L} \Gamma, \mathcal{R} \Gamma)$ be arbitrary. Since $y \Omega \subset K_{y} \Omega$ for some constant $K_{y}>0$, one has $\omega\left(y^{*} \cdot y\right) \leq K_{y}^{2} \tau(\cdot)$ both on $\mathcal{L} \Gamma$ and on $\mathcal{R} \Gamma$. Therefore, the GNS representation $\pi_{\omega}$ of $\omega$ is binormal on $C^{*}(\mathcal{L} \Gamma, \mathcal{R} \Gamma)$. Moreover, since $\mathbb{K}_{\mathcal{L} \Gamma} \cap C^{*}(\mathcal{L} \Gamma, \mathcal{R} \Gamma) \subset$ $\operatorname{ker} \pi_{\omega}$, the $*$-homomorphism from $C_{\lambda}^{*} \Gamma \otimes_{\text {alg }} C_{\rho}^{*} \Gamma$ into $\pi_{\omega}\left(C^{*}(\mathcal{L} \Gamma, \mathcal{R} \Gamma)\right)$ is continuous w.r.t. the minimal tensor norm. It follows from Lemma 9.2.9 in [BO] that the $*$-homomorphism from $\mathcal{L} \Gamma \otimes_{\text {alg }} \mathcal{R} \Gamma$ into $\pi_{\omega}\left(C^{*}(\mathcal{L} \Gamma, \mathcal{R} \Gamma)\right)$ is continuous w.r.t. the minimal tensor norm, too. Because of simplicity of $\mathcal{L} \Gamma$, this means that $\pi_{\omega}\left(C^{*}(\mathcal{L} \Gamma, \mathcal{R} \Gamma)\right)=\mathcal{L} \Gamma \otimes_{\min } \mathcal{R} \Gamma$, or 
equivalently that $\operatorname{ker} \pi_{\omega}=\operatorname{ker} \pi$. Therefore, $\mathbb{K}_{\mathcal{L} \Gamma} \cap C^{*}(\mathcal{L} \Gamma, \mathcal{R} \Gamma) \subset \operatorname{ker} \pi$. On the other hand, if $x \geq 0$ and $x \notin \mathbb{K}_{\mathcal{L} \Gamma}$, then there is a normalized weakly null sequence $\left(\xi_{n}\right)$ in $\Omega$ such that $\omega\left(x^{2}\right)=\operatorname{Lim}\left\|x \xi_{n}\right\|^{2}>0$ and a fortiori $x \notin \operatorname{ker} \pi_{\omega}$.

It follows that $\mathbb{K}\left(\ell^{2} \Gamma\right) \subset \operatorname{ker} \pi \subset \mathbb{K}_{\mathcal{L} \Gamma}$. The first inclusion is strict. Indeed, it is not hard to show that ker $\pi$ is non-separable. It is likely that the second is strict as well.

Recall that a finite von Neumann algebra $N$ has the property $(\Gamma)$ if there is a sequence $\left(u_{n}\right)$ of unitary elements in $N$ such that $u_{n} \rightarrow 0$ ultraweakly and $\left[u_{n}, a\right] \rightarrow 0$ ultrastrongly for every $a \in N$. We observe the following: Let $M \subset \mathbb{B}\left(L^{2}(M)\right)$ be a finite von Neumann algebra and $N \subset M$ be a von Neumann subalgebra with the property $(\Gamma)$. Then, one has $\mathbb{K}_{M} \cap C^{*}\left(N, M^{\prime}\right)=\{0\}$. Indeed, if $\left(u_{n}\right)$ is as above, then on the one hand $u_{n}^{*} x u_{n} \rightarrow x$ for every $x \in C^{*}\left(N, M^{\prime}\right)$, but on the other hand $u_{n}^{*} x u_{n} \rightarrow 0$ for every $x \in \mathbb{K}_{M}$. This observation, combined with the above theorem, implies the main theorem of Oz1]: A von Neumann subalgebra of $\mathcal{L} \Gamma$ which has the property $(\Gamma)$ is necessarily amenable.

5. Boundary of free group factors. Let $\mathbb{F}_{r}$ be the free group of rank $r \in \mathbb{N}$. For each $t \in \mathbb{F}_{r}$, we define $\chi_{t} \in \ell^{\infty} \mathbb{F}_{r}$ to be the characteristic function of the set of those elements in $\mathbb{F}_{r}$ whose last segments in the reduced forms are $t$. Let

$$
A=C^{*}\left(\left\{\chi_{t}: t \in \mathbb{F}_{r}\right\}\right) \subset \ell^{\infty} \mathbb{F}_{r}
$$

and observe that $\left[A, C_{\lambda}^{*} \mathbb{F}_{r}\right] \subset \mathbb{K}\left(\ell^{2} \mathbb{F}_{r}\right)$. Indeed, $\lambda(s) \chi_{t} \lambda(s)^{*} \delta_{x}=\chi_{t} \delta_{x}$ if $|x| \geq|s|+|t|$, and hence $\left[\chi_{t}, \lambda(s)\right]$ has finite rank. It is well-known that $B:=C^{*}\left(A, \rho\left(\mathbb{F}_{r}\right)\right) \cong A \rtimes \mathbb{F}_{r}$ is nuclear. Akemann and Ostrand's theorem stating that $\mathbb{F}_{r}$ is in the class $\mathcal{S}$ follows from this and

$$
\left[C_{\lambda}^{*} \mathbb{F}_{r}, B\right] \subset \operatorname{norm}-\operatorname{cl}\left(C_{\lambda}^{*} \mathbb{F}_{r} \cdot\left[C_{\lambda}^{*} \mathbb{F}_{r}, A\right]\right) \subset \mathbb{K}\left(\ell^{2} \mathbb{F}_{r}\right) .
$$

It would be interesting to know whether a similar fact holds true at the level of von Neumann algebras. Namely,

Problem. Is it true that $\left[A, \mathcal{L} \mathbb{F}_{r}\right] \subset \mathbb{K}_{\mathcal{L F}_{r}}$ ?

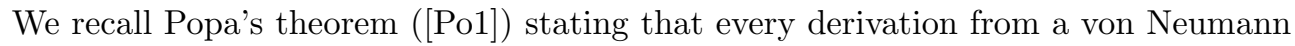
algebra $M \subset \mathbb{B}(\mathcal{H})$ into $\mathbb{K}(\mathcal{H})$ is inner. In particular, $[x, M] \subset \mathbb{K}(\mathcal{H})$ only if $x \in \mathbb{K}(\mathcal{H})+$ $M^{\prime}$. Nevertheless, the above problem has a positive answer if $r=1$, i.e., if $\mathbb{F}_{r}=\mathbb{Z}$. Indeed, let $\chi=\chi_{n \geq 0}$ for simplicity. Then, the projection $\chi$ is the Riesz projection which is bounded on $L^{4}(\widehat{\mathbb{Z}})$ (or on any $L^{q}$ with $1<q<\infty$, see Ga]). It follows from the Hölder inequality that for every $a \in \mathcal{L}(\mathbb{Z}) \cong L^{\infty}(\widehat{\mathbb{Z}})$, one has

$$
\|[\chi, a]\|_{\Omega} \leq\|\chi\|_{2,4}\|a\|_{4, \infty}+\|a\|_{2,4}\|\chi\|_{4, \infty} \leq C\|a\|_{L^{4}(\widehat{\mathbb{Z}})},
$$

where $\|\cdot\|_{p, q}$ stands for the operator norm from $L^{q}(\widehat{\mathbb{Z}})$ into $L^{p}(\widehat{\mathbb{Z}})$. Since $C_{\lambda}^{*} \mathbb{Z}$ is dense in $\mathcal{L} \mathbb{Z}$ w.r.t. the $L^{4}$-norm and $\left[\chi, C_{\lambda}^{*} \mathbb{Z}\right] \in \mathbb{K}\left(\ell^{2} \mathbb{Z}\right)$, one obtains that $[\chi, \mathcal{L} \mathbb{Z}] \in \mathbb{K}_{\mathcal{L} \mathbb{Z}}$. The author is unable to extend this argument to $\mathbb{F}_{r}$ with $r \geq 2$, because he does not know whether the 'Riesz projection' on $\mathbb{F}_{r}$ is a bounded operator from $\mathcal{L} \mathbb{F}_{r}$ into $L^{4}\left(\mathcal{L} \mathbb{F}_{r}\right)$.

Acknowledgments. The author was supported by JSPS. 


\section{References}

[AO] C. A. Akemann and P. A. Ostrand, On a tensor product $C^{*}$-algebra associated with the free group on two generators, J. Math. Soc. Japan 27 (1975), 589-599.

[BO] N. P. Brown and N. Ozawa, $C^{*}$-algebras and Finite-Dimensional Approximations, Grad. Stud. Math. 88, Amer. Math. Soc., Providence, RI, 2008.

[Ga] J. Garnett, Bounded Analytic Functions, revised first edition, Graduate Texts in Mathematics 236, Springer, New York, 2007.

[Ma] B. Magajna, A topology for operator modules over $W^{*}$-algebras, J. Funct. Anal. 154 (1998), 17-41.

[Oz1] N. Ozawa, Solid von Neumann algebras, Acta Math. 192 (2004), 111-117.

[Oz2] N. Ozawa, A Kurosh-type theorem for type $\mathrm{II}_{1}$ factors, Int. Math. Res. Not. 2006, Art. ID 97560, $21 \mathrm{pp}$.

[Pe] J. Peterson, $L^{2}$-rigidity in von Neumann algebras, Invent. Math. 175 (2009), 417-433.

[Pi] G. Pisier, Factorization of Linear Operators and Geometry of Banach Spaces, CBMS Regional Conf. Series in Math. 60, Amer. Math. Soc., Providence, RI, 1986.

[Po1] S. Popa, The commutant modulo the set of compact operators of a von Neumann algebra, J. Funct. Anal. 71 (1987), 393-408.

[Po2] S. Popa, On Ozawa's property for free group factors, Int. Math. Res. Not. 2007, Art. ID rnm036, $10 \mathrm{pp}$.

[Ta] M. Takesaki, Theory of Operator Algebras. II, Encyclopaedia of Mathematical Sciences 125, Springer, Berlin, 2003. 
